\author{
David M. Greer \\ Franco Valenza \\ Giuseppe Citerio
}

\section{Improving donor management and transplantation success: more research is needed}

Received: 7 January 2015

Accepted: 11 January 2015

Published online: 27 January 2015

(C) Springer-Verlag Berlin Heidelberg and ESICM 2015

D. M. Greer $(\bullet)$

Department of Neurology, Yale University School of Medicine, LLCI 912, Chairs Office, 15 York Street, New Haven CT 06520, USA

e-mail: david.greer@yale.edu

F. Valenza

Department of Pathophysiology and Transplantation, University of Milan, Milan, Italy

G. Citerio

Department of Health Science, University of Milan Bicocca, Monza, Italy

At the time of writing, million of patients worldwide are on a waiting list for a lifesaving organ transplant, 123,833 in the United States [1] and nearly 63,000 in the European Union [2]. However, the number of organs that become available each year falls far short of providing for those in need, and thousands die each year waiting for transplantation. There are clearly many missed opportunities in the severely brain-injured population, many patients dying without the care teams pursuing the chance to give life to others through donation $[3,4]$.

Donation after brain death (BD) still remains the main source for organs, and it carries the advantage of being performed in a relatively controlled setting with ongoing perfusion and oxygenation. Death as determined by neurological criteria has been accepted worldwide $[5,6]$, and strict criteria exist for proper determination [7]. However, delays in the diagnosis of $\mathrm{BD}$ have been associated with decreased rates of organ donation, as well as decreased viability of organs transplanted [8]. One of the obstacles is the inherent difficulty that families have dealing with these highly stressful situations, in which they are trying to balance the desire to be altruistic with their need to grieve the often sudden loss of their loved one [9].

The reasons for decreased organ viability are multiple, likely related to secondary effects from the occurrence of BD itself. BD induces a pro-inflammatory state with a massive catecholamine release with ensuing hormonal, metabolic and hemodynamic effects. BD has been associated with renal injury [10]. Hormonal effects include decreases in serum cortisol, insulin, thyroid hormone and other pituitary hormone levels, and non-randomized studies have suggested an improvement in hemodynamic status with hormone replacement [11]. This has not been confirmed in randomized controlled trials (RCT), and the evidence for their use is poor.

Hand in hand with the hormonal alterations is the impairment in the hemodynamic system, affecting organ perfusion and sometimes even leading to cardiac arrest [12]. Hemodynamic instability following BD is frequent and can usually be attributed to multiple causes, as a sympathetic surge preceding medullary damage in BD leading to a cardiac damage, a spinal cord infarction resulting in loss of sympathetic tone and further hypotension, and pituitary dysfunction and consequent diabetes insipidus. Therefore, many guidelines and protocols aim at repairing hormonal imbalances and maintaining hemodynamic stability [13, 14], although strong data to support this practice are lacking. Physicians and organ procurement organizations (OPO) have thus based their practices on hypotheses and non-randomized clinical data. There has been a call to arms for improved donor management that will be evidence-based and effective [15].

In an article recently published in Intensive Care Medicine, Al-Khafaji et al. [16] reported the results of the Monitoring Organ Donors to Improve Transplantation 


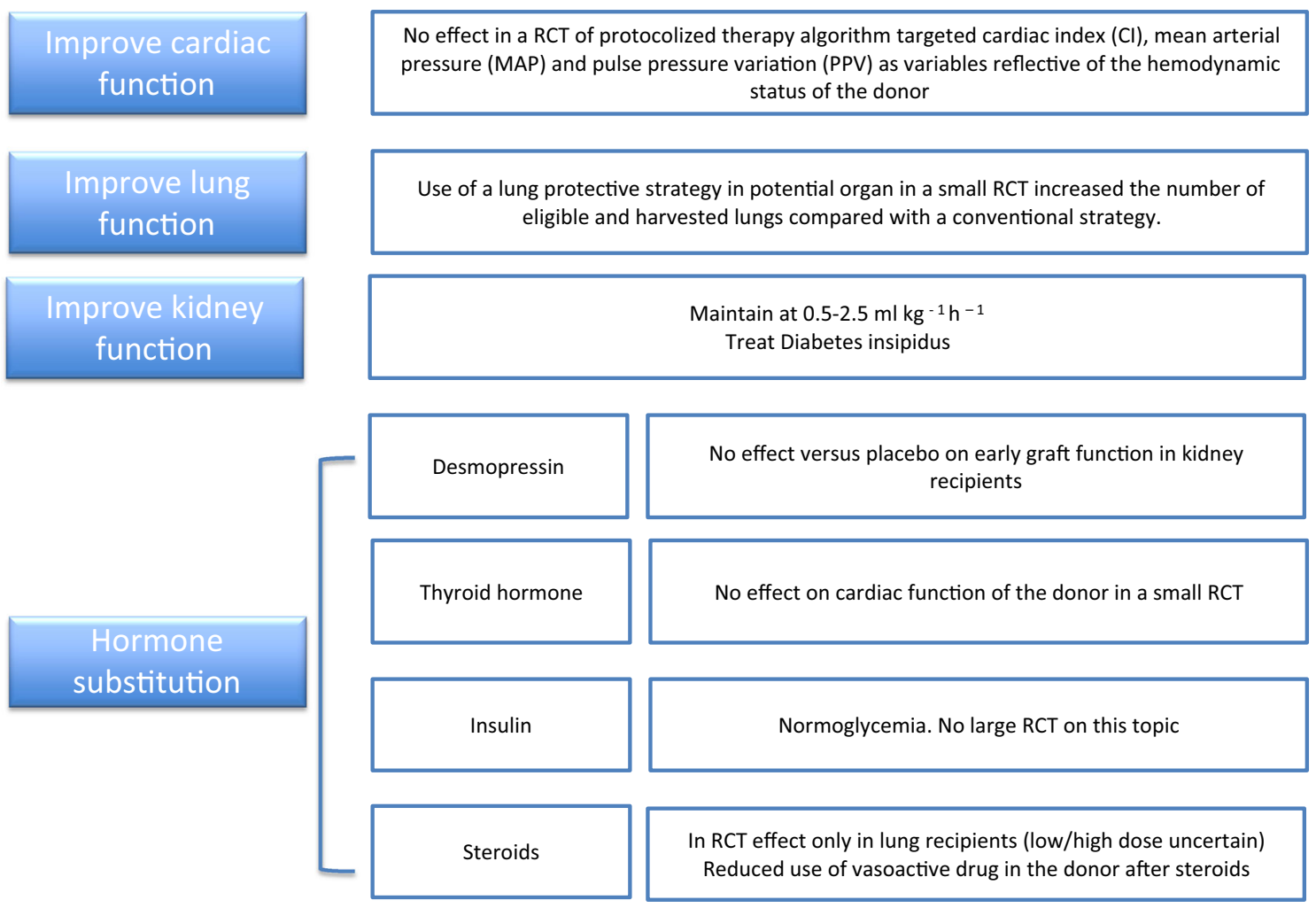

Fig. 1 Summary of the treatment aims in the potential organ donor and the evidence coming from RCT. For most of the targets, there is no available guidance or negative guidance from RCT, and most are small RCT

Results (MOnIToR) trial, a protocolized fluid therapy in brain-dead organ donors in an effort to improve organ viability and survival in recipients. Using eight organ procurement organizations, they randomly assigned donors to either protocolized or usual care; the protocolized therapy algorithm targeted cardiac index (CI), mean arterial pressure (MAP) and pulse pressure variation (PPV) as variables reflective of the hemodynamic status of the donor, and whether fluid therapy was warranted. The protocol was ultimately implemented in $76 \%$ of those randomized to the interventional arm. In the end, there was no statistically significant difference between the arms: 3.39 organs per donor with protocolized care, versus 3.29 with usual care. In a modified intention-totreat analysis, there remained no significant difference between the groups. The mortality rate at 1 year was the same between the groups ( 7.8 vs. $7.9 \%)$.

At first glance, there appears to be little positive that can be taken from this trial. But it is important to recognize the impacts of the trial, as well as its limitations, that may open the door for future trials. First and foremost, the trial confirms the possibility of conducting large, good RCT in the field of organ donation that so badly needs clinical data. Without such trials, we cannot truly know whether our current interventions actually work, or might be detrimental to donor management and organ viability. Secondly, the authors performed a "responder analysis," in which the donors in the protocolized arm were deemed to have responded to the fluid protocol and were analyzed separately; this subgroup analysis showed a nearly significant difference in the number of organs transplanted per donor, an increase of $0.58 \pm 2.07$ (95\% CI 0.02-1.19, $p=0.059$ ). Although subgroup analyses are not "take-home messages," they do give an indication for the right type of groups to study in the future as a priori hypotheses.

The limitations of the trial are notable. The choice of hemodynamic parameters was limited to only CI, MAP and PPV, and, although these are reasonable choices, there may be other factors that might guide fluid management more accurately or appropriately, such as the use of pulmonary arterial catheters with other hemodynamic markers. The device used to measure CI, MAP and PPV was complicated, requiring education and training at multiple sites. Obviously, critical care units are adept and comfortable adapting to new technology, but this device appears to have been particularly troublesome, with zero participants classifying the device as "easy" to use. The protocol was implemented in $76 \%$ of the subjects randomized to intervention, meaning that $24 \%$ never 
received the intervention, and only a third of the coordinators strictly adhered to the protocol. These numbers are quite concerning for a randomized controlled trial.

One also needs to look at the control group, who received "usual care." Usual care may have been quite variable in this trial, with differences in the types of fluids administered, vasopressors/antihypertensive agents received, and other aspects of care of the donor. The authors justify this by stating this usual care "will reflect the care currently being provided at sites" [17]. However, the trial would have been greatly strengthened by settling on one approach that could be used at all sites, especially considering how care in the protocolized arm was strictly specified. The problem we face is that we do not have uniform standard care, accepted by most centers.

The choice of outcomes appears to be appropriate, although others could have been considered. Rather than looking simply at the number of organs transplanted per donor, a variable that is likely dependent on a number of factors, perhaps a more meaningful primary outcome measure would have been the viability of the organs at 6 or 12 months; particularly interesting would be to look at this outcome by type of organ transplanted. Mortality is a measure that gets at this question, but is also affected by many factors outside of the transplant. Mortality is clearly a relevant outcome, well addressed by RCT trials such as the one by presented by Al-Khafaji [16].
The MOnIToR trial opens the door for future trials studying both hemodynamic and other interventions that may improve donor management and transplantation success. Ventilation approaches using lung protective strategies have shown great promise in lung transplantation [18]. Hypothermia may be a useful intervention to decrease the detrimental effects of hypoxia and ischemia [19]. Extracorporeal membrane oxygenation (ECMO) has been the recent subject of study in both non-heart beating and brain-dead donors, and also appears promising [20]. Furthermore, a number of different interventions in the post-harvested organs may make significant impacts in organ viability, including ex vivo lung perfusion, different preservative solutions, and mechanical perfusion [21]. RCT have to run in parallel with these smaller investigations, exploring pathophysiological mechanisms or testing hypotheses (Fig. 1).

As Doig wrote [22], information generated by randomized controlled trials has helped to save tens of thousands of lives over the past 40 years, and the appropriate use of objective research evidence, has to play a major role in improving donor management and transplantation success.

Conflicts of interest The authors declare no conflict of interest for this manuscript.

\section{References}

1. http://www.optn.transplant.hrsa.gov

2. https://urldefense.proofpoint.com/v2/ $\mathrm{url} ? \mathrm{u}=\mathrm{http}-3 \mathrm{~A} \_$ec.europa.eu_ health_blood-5Ftissues-5Forgans docs_ev-5F20131007-5Frd3-5Fen. pdf \&d $=$ AwIGaQ\&c $=-\operatorname{dg} 2 \mathrm{~m} 7 \mathrm{z}$ WuuDZ0MUcV7Sdqw\&r = ZWlAM66QyGQDUFjA7a RF8qvtFTY7GRC8GCEwe OghtyQ\&m = IloS2AyNSdb RNGxprUhVfTA7HJrEsrZXd hpZ2hWDAmU\&s $=7 \mathrm{f} 7 \mathrm{wSOJge}$ uMYYYWR0O9dVUK8V2cZIf_AYTeyoWc_I\&e=

3. Lesieur O, Leloup M, Gonzalez F, Mamzer MF (2014) Eligibility for organ donation following end-of-life decisions: a study performed in 43 French intensive care units. Intensive Care Med 40(9):1323-1331

4. Kutsogiannis DJ, Asthana S, Townsend DR, Singh G, Karvellas CJ (2013) The incidence of potential missed organ donors in intensive care units and emergency rooms: a retrospective cohort. Intensive Care Med 39(8):1452-1459
5. Wijdicks EF (2002) Brain death worldwide: accepted fact but no global consensus in diagnostic criteria. Neurology 58(1):20-25

6. Shemie SD, Hornby L, Baker A et al (2014) International guideline development for the determination of death. Intensive Care Med 40(6):788-797

7. Wijdicks EF, Varelas PN, Gronseth GS, Greer DM (2010) American Academy of N. Evidence-based guideline update: determining brain death in adults: report of the Quality Standards Subcommittee of the American Academy of Neurology. Neurology 74(23):1911-1918

8. Lustbader D, O'Hara D, Wijdicks EF et al (2011) Second brain death examination may negatively affect organ donation. Neurology 76(2):119-124

9. Shaw D, Elger B (2014) Persuading bereaved families to permit organ donation. Intensive Care Med 40(1):96-98

10. Westendorp WH, Leuvenink HG, Ploeg RJ (2011) Brain death induced renal injury. Curr Opin Organ Transplant 16(2):151-156
11. Rech TH, Moraes RB, Crispim D, Czepielewski MA, Leitao CB (2013) Management of the brain-dead organ donor: a systematic review and metaanalysis. Transplantation 95(7):966-974

12. Bugge JF (2009) Brain death and its implications for management of the potential organ donor. Acta Anaesthesiol Scand 53(10):1239-1250

13. Westphal GA, Caldeira Filho M, Vieira KD et al (2011) Guidelines for potential multiple organ donors (adult): part II. Mechanical ventilation, endocrine metabolic management, hematological and infectious aspects. Rev Bras Ter Intensiva 23(3):269-282

14. Rosendale JD, Chabalewski FL, McBride MA et al (2002) Increased transplanted organs from the use of a standardized donor management protocol. Am J Transplant 2(8):761-768

15. Al-Khafaji A, Murugan R, Kellum JA (2013) What's new in organ donation: better care of the dead for the living. Intensive Care Med 39(11):2031-2033 
16. Al-Khafaji A et al (2015) Protocolized fluid therapy in brain-dead donors: the multicenter randomized MOnIToR trial. Intensive Care Med. doi: 10.1007/s00134-014-3621-0

17. Al-Khafaji A, Murugan R, Wahed AS et al (2013) Monitoring Organ Donors to Improve Transplantation Results (MOnIToR) trial methodology. Crit Care Resusc 15(3):234-240
18. Randell T, Orko R, Hockerstedt K (1990) Peroperative fluid management of the brain-dead multiorgan donor. Acta Anaesthesiol Scand 34(7):592-595

19. Moers C, Smits JM, Maathuis MH et al (2009) Machine perfusion or cold storage in deceased-donor kidney transplantation. N Engl J Med 360(1):7-19
20. Englesbe MJ, Woodrum D, Debroy M et al (2004) Salvage of an unstable brain dead donor with prompt extracorporeal support. Transplantation 78(12): 1815

21. Goldstein MJ, Lubezky N, Yushkov Y, Bae C, Guarrera JV (2012) Innovations in organ donation. Mt Sinai $\mathrm{J}$ Med 79(3):351-364

22. Doig GS, Roberts I, Bellomo R. The tens of thousands of lives saved by randomized clinical trials in critical care. Intensive care Med. 2014: 1-4 